\title{
Proposals for the generation of angular momentum from non-uniformly polarized beams
}

\author{
Mara Alonso, Gemma Piquero, Julio Serna* \\ Departamento de Óptica, Universidad Complutense de Madrid, 28040 Madrid, Spain
}

\section{A R T I C L E I N F O}

\section{Article history:}

Received 19 July 2011

Received in revised form 19 November 2011

Accepted 28 November 2011

Available online 11 December 2011

Keywords:

Physical optics

Polarization

Beam characterization

Angular momentum

\begin{abstract}
A B S T R A C T
Several optical arrangements using non-uniformly polarized fields are proposed for generating beams with spin and/or orbital angular momentum. By choosing adequately the input beam polarization and the characteristics of the different proposed set-ups we can control the overall angular momentum of the output beam at will. The orbital angular momentum is analyzed with the beam moments theory and the spin term is evaluated using the averaged $s_{3}$ Stokes parameter.
\end{abstract}

(C) 2011 Elsevier B.V. All rights reserved.

\section{Introduction}

In the last years angular momentum has attracted growing attention as a consequence of its use in applications such as optical tweezers, information encoding for optical communications and quantum computation, or novel techniques in astronomical observations among others (see for example Refs. [1-6]).

As is well known, a monochromatic light beam propagating along the $z$ direction can transport not only linear but also angular momentum. It is found that angular momentum splits naturally in two components: an intrinsic (or spin) part related to polarization, and an extrinsic component, also called orbital angular momentum, associated with the optical phase in coherent beams.

The generation of spin and orbital angular momentum is a subject of increasing interest because it can provide insight into the fundamental properties of light and its interaction with matter. In some papers different schemes to produce and detect spin and/or orbital angular momentum have been proposed. For example Marrucci et al. proposed to use "q-plates" (inhomogeneous birrefringent elements) or inhomogeneous liquid crystals $[7,8]$. They experimentally demonstrated the spin-orbital angular momentum transfer detecting patterns of light with helical modes using an interferometer. Zhao et al. proposed to use a circularly polarized Laguerre-Gaussian laser beam and studied the transfer from

\footnotetext{
* Corresponding author
}

E-mail addresses: mara.alonso.l@gmail.com (M. Alonso), piquero@fis.ucm.es (G. Piquero), azul@fis.ucm.es (J. Serna). spin to orbital angular momentum when the beam is tightly focused by means of a convergent lens [9]. They verified the generation of a beam with orbital angular moment by controlling the rotation of absorptive particles. Some other papers [10-13] have considered inhomogeneously polarized fields with vortices and have studied the averaged and local angular momentum.

Another important issue is the evaluation of the angular momentum. The spatial structure of laser beams can be characterized by means of global parameters based on the so-called irradiance moments [14-17]. It has been demonstrated that some second-order irradiance beam moments are closely connected to the orbital angular momentum of a beam, and are very useful in order to quantify it. Expressions for the angular momentum content of partially coherent beams and non-uniformly polarized beams provided by this formalism have been published in the literature $[18,19]$. On the other hand, the spin contribution can be calculated using the Stokes parameters.

In this paper we propose different arrangements to endow a beam with global spin and/or global orbital angular moment by using uniformly and non uniformly polarized beams [20]. In order to synthesize this type of fields the use of some devices such as unconventional polarizers and polarization converters is proposed. The evaluation of the global angular momentum will be done with the beam moments theory and the averaged $s_{3}$ Stokes parameter.

The paper is structured as follows: after the Introduction given in Section 1, we use Section 2 to present the formalism and the optical systems that we are going to apply. In Section 3 different proposed procedures to generate spin and/or orbital angular momentum are described and analyzed. Finally, Section 4 summarizes the main conclusions of this work. 


\section{Formalism and unconventional optical elements}

Let us consider a quasimonocromatic field with mean angular frequency $\omega$, propagating along the $z$-axis within the paraxial approximation, and with a non uniform distribution of polarization across the beam profile. By using the Jones formalism we can write the field as

$\mathbf{E}(\mathbf{r})=\left(\begin{array}{c}E_{s}(\mathbf{r}) \\ E_{p}(\mathbf{r})\end{array}\right)$

where $\mathbf{r}=(r, \theta)$ are polar coordinates and $E_{s}, E_{p}$ are the field components orthogonal to the propagation direction. As it has been shown in a previous work [19], for non uniformly polarized beams the time averaged angular momentum density flux propagating through a $z$ plane is separated in orbital $(L)$ and polarization contributions $(S)$,

$\bar{J}_{z}=\bar{J}_{z}^{L}+\bar{J}_{z}^{S}$

Within this formalism the orbital angular momentum term is divided into $s$ and $p$ components as

$\bar{J}_{z}^{L}=\frac{P_{s}}{c}\left(\langle x v\rangle_{s s}-\langle y u\rangle_{s s}\right)+\frac{P_{p}}{c}\left(\langle x v\rangle_{p p}-\langle y u\rangle_{p p}\right)$,

being $P_{i}$, with $i=s, p$, the power of each component, and

$\langle x v\rangle_{i i}=\frac{\epsilon_{0} c}{2 k P_{i}} \iint x \operatorname{Im}\left(E_{i}^{*} \frac{\partial E_{i}}{\partial y}\right) d x d y$,

$\langle y u\rangle_{i i}=\frac{\epsilon_{0} c}{2 k P_{i}} \iint y \operatorname{Im}\left(E_{i}^{*} \frac{\partial E_{i}}{\partial x}\right) d x d y$,

the crossed irradiance moments, also for each component. In those formulas $\mathrm{c}$ is the speed of light, $\epsilon_{0}$ is the vacuum permitivity and $k=\omega / c$ is the wave vector modulus. Note that if the beam has an $e^{i n \theta}$ factor there is a contribution to the orbital term of the angular momentum. On the other hand, the spin contribution, $\bar{J}_{z}^{S}$, is given by

$\bar{J}_{z}^{S}=\epsilon_{0} c \operatorname{Im} \iint E_{s}^{*} E_{p} d x d y=\frac{1}{\omega} \hat{s}_{3}$,

where $\hat{s}_{3}$ represents the average of the $s_{3}$ Stokes parameter over the whole transverse section of the beam. This means that the beam must have some global circular polarization content in order to have a spin contribution to the angular momentum.

As can be seen from Eqs. (2)-(6), angular momentum can be obtained in an easy way. Orbital angular momentum can be calculated from the beam irradiance moments, while beam polarization affects the spin term.

We can also define the angular momentum per photon, $j$,

$j=\bar{J}_{z} \frac{\hbar \omega}{P}$,

with its orbital and spin components,

$j^{L}=k \frac{P_{s}}{P}\left(\langle x v\rangle_{s s}-\langle y u\rangle_{s S}\right) \hbar+k \frac{P_{p}}{P}\left(\langle x v\rangle_{p p}-\langle y u\rangle_{p p}\right) \hbar$,

$j^{S}=\frac{\hat{s}_{3}}{P} \hbar$,

where we have used that the photon flux (photons per unit time) is $P / \hbar \omega$, $P$ being the total power of the beam, $P=P_{s}+P_{p}$, and $\hbar$ being the normalized Planck's constant.

We will now proceed to detail the unconventional optical elements which we propose to use in order to provide our beam with angular momentum.

\subsection{Spiral phase plates (SPP)}

The first element considered is the spiral phase plate (SPP), which adds a $e^{i n \theta}$ term to the beam phase. A SPP can be used to generate a vortex in a beam, and can even be designed to introduce a fractional number in the topological charge of the beam (see for example Refs. $[21,22])$.

It should be noted that with the term SPP we consider all different optical systems used to generate the above mentioned phase singularity in a beam, such as plates made of birefringent elements, computer generated holograms, liquid crystal elements, etc. [23-27].

\subsection{Axis finder $(A F)$}

A second element that we are going to consider is the so-called azimuthal polarizer, or axis finder $(A F)$. This type of non-conventional polarizers have been used to synthesize special kinds of nonuniformly polarized fields, in particular spirally polarized beams [28]. This optical device can be represented by its Jones matrix,

$A F=\left(\begin{array}{cc}\sin ^{2} \theta & -\cos \theta \sin \theta \\ -\cos \theta \sin \theta & \cos ^{2} \theta\end{array}\right)$.

By means of an $A F$ we can obtain a beam whose state of polarization is azimuthal across the beam section [28]. Note that in this case, if we want to have the same irradiance distribution as at the input, we need a circularly polarized incident beam. An example of this system is the Polarization Axis Finder Model 10901A from TSI.

\subsection{Polarization converter $(P C)$}

Finally, the third element considered is the so called polarization converter $(P C)$, whose Jones matrix for incident light with linear polarization along $x$ or $y$ axis is given by [29]

$P C=\left(\begin{array}{cc}\cos \theta & -\sin \theta \\ \sin \theta & \cos \theta\end{array}\right)$.

When a linearly polarized beam with horizontal or vertical polarization propagates through the $P C$ a radially or an azimuthally polarized beam is obtained, respectively. A PC can also be used to generate spirally polarized beams [28]. An example of a polarization converter is the Radial-Azimuthal Polarization Converter from Arcoptix.

\section{Arrangements and results}

In this section we describe and compare different procedures for generating spin and orbital angular momentum. In particular, we will consider four types of arrangements. Note that all these proposed systems can also be combined in several ways.

Regarding polarization, in the first set-up we use as input (and obtain as output) uniformly and totally polarized beams in order to simplify, but the set-up could be used with non uniformly polarized fields too. In the second and third arrangements we start from totally and uniformly polarized initial beams and generate beams with non uniform polarization across their transverse sections. Finally, in the fourth proposed system we use initial uniformly and totally polarized beams but the output beam will be a uniformly or non uniformly polarized field depending on the elements considered.

On the other hand, for simplicity, we will assume in all the arrangements that the incident beam has no initial orbital angular momentum. In a general case, depending on the functional form of its amplitude, the input beam could have or not have an initial orbital angular momentum, and by means of the proposed arrangements angular momentum could be added or subtracted. 


\subsection{Spiral phase plate}

This first setting has been described in Ref. [19], and is a very usual form to generate angular momentum. In this set-up, described in Fig. 1, a uniformly and totally polarized beam $\mathbf{E}_{i}(\mathbf{r})$ (in the following subindex $i$ refers to the input beam) is considered to propagate through a spiral phase plate and a constant phase plate. The initial beam is linearly polarized at $45^{\circ}$ respect to the plane of incidence,

$\mathbf{E}_{i}(\mathbf{r})=\frac{f(\mathbf{r})}{\sqrt{2}}\left(\begin{array}{l}1 \\ 1\end{array}\right)$

where $f(\mathbf{r})$ is a function that represents the amplitude and could be complex, in general. The spin component of this beam is $j_{i}^{S}=0$.

After propagation through the spiral phase plate SPP and the constant phase plate $P P_{\delta}$ we obtain (subindex $o$ denotes the output beam)

$\mathbf{E}_{o}(\mathbf{r})=\frac{f(\mathbf{r})}{\sqrt{2}} e^{i n \theta}\left(\begin{array}{c}1 \\ e^{i \delta}\end{array}\right)$.

Here and in the following we are going to drop the common phase factors. From Eq. (13) we see that with this arrangement $P P_{\delta}$ adds a constant phase factor to one component and $e^{i n \theta}, \theta$ being the azimuthal angle, is the helical phase added by means of SPP, which represents a single vortex of charge $n$. This beam is uniformly polarized across its transverse section, and its angular momentum components, given by Eqs. (8)-(9), are

$j_{0}^{L}=n \hbar$,

$j_{0}^{S}=\sin \delta \hbar$,

where the first term corresponds to the orbital angular momentum while the second is the polarization contribution. Note that with this set-up the spin contribution is obtained due only to the use of the constant phase plate, while the orbital contribution comes only from spiral phase plate, with no crossed terms.

\subsection{Axis finder}

The second proposed configuration uses a quarter wave phase plate $P P_{\lambda / 4}$ and an $A F$, as shown in Fig. 2.

Let us begin considering a uniformly and linearly polarized beam with the Jones vector given by

$\mathbf{E}_{i}(\mathbf{r})=\frac{f(\mathbf{r})}{\sqrt{2}}\left(\begin{array}{l}1 \\ 1\end{array}\right)$

From this equation it is clear that $j_{i}^{S}=0$. If the input beam passes through a quarter wave phase plate with the axis at $0^{\circ}$ or $90^{\circ}$ respect to the $x$ direction, we obtain a circularly uniformly polarized beam

$\mathbf{E}_{\lambda / 4}(\mathbf{r})=\frac{f(\mathbf{r})}{\sqrt{2}}\left(\begin{array}{c}1 \\ \pm i\end{array}\right)$,

where the sign indicates if it is a right or a left-handed circularly polarized beam. Now we have $j_{\lambda / 4}^{S}= \pm \hbar$, so the quarter wave phase

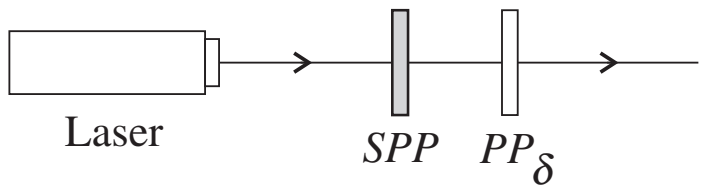

Fig. 1. Arrangement with spiral phase plate $S P P$ and a constant phase plate $P P_{\delta}$.

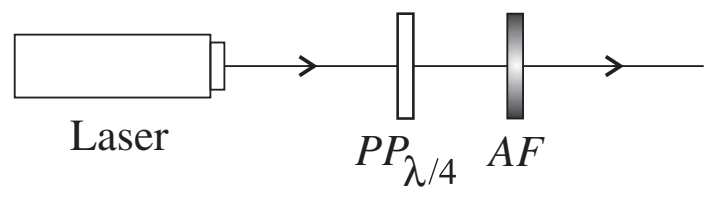

Fig. 2. Arrangement using a quarter wave phase plate $P P_{\lambda / 4}$ and an axis finder $A F$.

plate has added spin angular momentum. But we have the same orbital angular momentum as the input beam, since with a quarter wave phase plate no additional $e^{i n \theta}$ factor is added.

The circularly polarized beam is now propagated through an $A F$. By using Eq. (10) the output field

$\mathbf{E}_{o}(\mathbf{r})=\frac{f(\mathbf{r})}{\sqrt{2}} e^{ \pm i(\theta-\pi / 2)}\left(\begin{array}{c}\sin \theta \\ -\cos \theta\end{array}\right)$,

is obtained.

Eq. (18) represents a linearly but non uniformly polarized beam in the transverse section, with the electric field vector oriented along the azimuthal direction. Consequently $j_{o}^{S}=0$. But now using Eqs. (4) and (5) we find a non zero orbital angular moment. For this beam the angular momentum orbital and spin components are

$j_{o}^{L}= \pm \hbar$,

$j_{0}^{S}=0$.

It should be noticed that we can add or subtract angular momentum to an input beam just by rotating $90^{\circ}$ the quarter wave phase plate. The final spin contribution is null, but we have used an intermediate spin contribution in order to obtain a gain or a loss of the orbital angular momentum depending on the orientation of the phase plate. Of course a non zero spin contribution could be added using an additional constant phase plate.

\subsection{Mach-Zehnder interferometer and spiral phase plate}

The third arrangement, suggested in Ref. [19], makes use of nonuniformly totally polarized (NUTP) beams. In order to synthesize the NUTP beam a Mach-Zehnder interferometer with polarized beam splitters is considered together with an additional $P P_{\delta}$ and $S P P$ as shown in Fig. 3. The input beam is a collimated and linearly polarized beam at $45^{\circ}$ respect to the plane of incidence. The polarizing beam splitter yields orthogonal polarizations at each arm of the interferometer, as shown in Fig. 3. By means of $P P_{\delta}$ and SPP we can

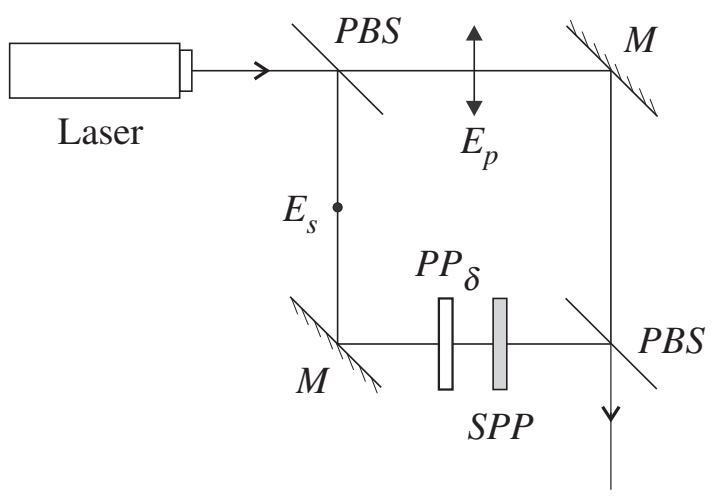

Fig. 3. Mach-Zehnder arrangement with spiral phase plate SPP and constant phase plate $P P_{\delta}$. $P B S$ are polarizing beam splitters and $M$ mirrors. 
introduce a constant phase factor $e^{i \delta}$ in the $E_{S}$ component and a vortex of charge $n$ respectively, obtaining at the output of the interferometer a field given by

$\mathbf{E}_{o}(\mathbf{r})=\frac{f(\mathbf{r})}{\sqrt{2}}\left(\begin{array}{c}e^{i n \theta} e^{i \delta} \\ 1\end{array}\right)$

Note that this beam has the same irradiance profile as the initial beam or the beam used in the first set-up (SPP), but now it is a nonuniformly polarized beam: the polarization vector depends on $\theta$, and therefore varies from point to point. The angular momentum components for this generated NUTP field are

$j_{o}^{L}=\frac{n}{2} \hbar$,

$j_{o}^{S}=\frac{\sin (n \pi) \sin (n \pi+\delta)}{n \pi} \hbar$.

In this case we have two contributions as in Eqs. (14)-(15), but now there is a crossed term and the spin term can be cancelled independently of the phase $\delta$ if $n$ is an integer. In this case by varying adequately the input beam, the phase of the $P P_{\delta}$ and the order of the SPP we can obtain different values of the angular momentum.

\subsection{Mach-Zehnder interferometer and polarization converters}

Finally, in this last setting we propose to introduce polarization converters $(P C)$ and constant phase plates at each arm of a MachZehnder interferometer, as shown in Figs. 4 and 5. As in the previous arrangement the input beam is a linearly and totally polarized collimated beam and the polarization at each arm is orthogonal thanks to a polarizing beam splitter. We will consider two particular cases. In the first one (Fig. 4) we have two polarization converters and a half wave phase plate. The second one (Fig. 5) is similar but two quarter wave phase plates are added, one at each arm.

Let us beginning with the set-up shown in Fig. 4. In this MachZehnder interferometer just before the $P C$ we only have $s$ component in the arm with no constant phase plate:

$\mathbf{E}_{1}(\mathbf{r})=\frac{f(\mathbf{r})}{\sqrt{2}}\left(\begin{array}{l}1 \\ 0\end{array}\right)$,

The $P C$ generates a radially polarized beam

$\mathbf{E}_{1}^{\prime}(\mathbf{r})=\frac{f(\mathbf{r})}{\sqrt{2}}\left(\begin{array}{c}\cos \theta \\ \sin \theta\end{array}\right)$,

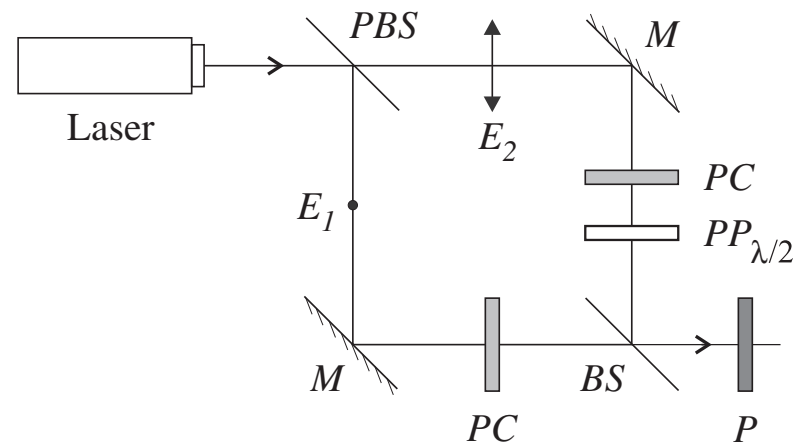

Fig. 4. Mach-Zehnder arrangement with polarization converters $P C$ and a half wave phase plate $P P_{\lambda / 2}$. $P B S$ is a polarizing beam splitter, $M$ mirrors, $B S$ a beam splitter and $P$ a polarizer.

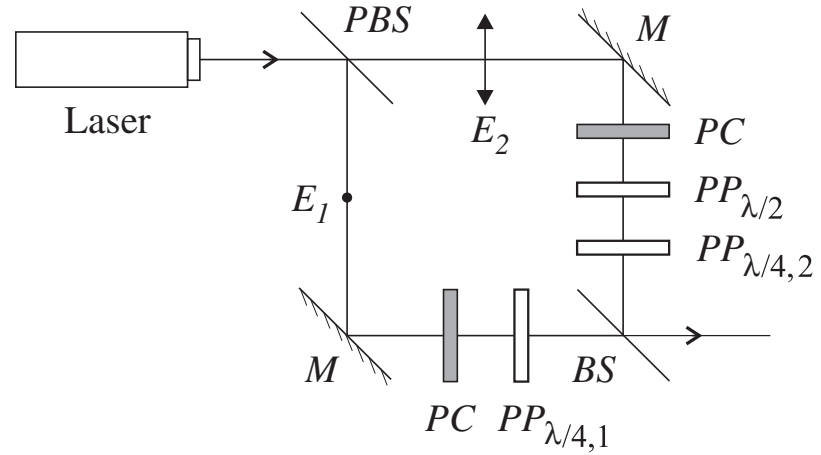

Fig. 5. Mach-Zehnder arrangement with polarization converters $P C$, a half wave phase plate $P P_{\lambda / 2}$ and quarter wave phase plates $P P_{\lambda / 4}$. $P B S$ is a polarizing beam splitter, $M$ mirrors and $B S$ a beam splitter.

where we have used Eq. (11). This is a beam with $j_{1}^{S}=0$ and $j_{1}^{L}=0$ (as in previous arrangements we will consider beams with no initial orbital angular momentum).

Due to the polarizing beam splitter the state of polarization in arm 2 is orthogonal to that in arm 1, and before the $P C$ we have

$\mathbf{E}_{2}(\mathbf{r})=\frac{f(\mathbf{r})}{\sqrt{2}}\left(\begin{array}{l}0 \\ 1\end{array}\right)$

The PC generates now an azimuthally polarized beam,

$\mathbf{E}_{2}^{\prime}(\mathbf{r})=\frac{f(\mathbf{r})}{\sqrt{2}}\left(\begin{array}{c}-\sin \theta \\ \cos \theta\end{array}\right)$

This beam propagates through a half wave phase plate described by the Jones matrix

$P P_{\lambda / 2}=\left(\begin{array}{cc}-i & 0 \\ 0 & i\end{array}\right)$

resulting in

$\mathbf{E}_{2}^{\prime \prime}(\mathbf{r})=i \frac{f(\mathbf{r})}{\sqrt{2}}\left(\begin{array}{c}\sin \theta \\ \cos \theta\end{array}\right)$

with no orbital or spin contribution.

When beams $\mathbf{E}_{1}^{\prime}(\mathbf{r})$ and $\mathbf{E}_{2}^{\prime \prime}(\mathbf{r})$ are recombined by means of an output beam splitter the resulting beam is

$E_{0}(\mathbf{r})=\frac{f(\mathbf{r})}{2}\left(\begin{array}{c}e^{i \theta} \\ i e^{-i \theta}\end{array}\right)$

This is a non uniformly and totally polarized beam with the same irradiance as the input beam and with no overall spin or orbital angular momentum also. But note that each component $s$ or $p$ of the beam gives a different contribution to the orbital angular momentum. This means that we could use a linear polarizer to control the orbital angular momentum. By placing a linear polarizer $P$ with its transmission axis oriented at an angle $\alpha$ at the output of the interferometer we have

$E_{o, P}(\mathbf{r})=\frac{f(\mathbf{r})}{2}\left(e^{i \theta} \cos \alpha+i e^{-i \theta} \sin \alpha\right)\left(\begin{array}{c}\cos \alpha \\ \sin \alpha\end{array}\right)$.

Now by rotating the polarizer it is possible to obtain an orbital angular momentum which varies between $+\hbar$ and $-\hbar$, resulting in

$j_{o}^{L}=\hbar \cos 2 \alpha$, 
$j_{0}^{S}=0$

where we have used that $j^{S}=0$ for linearly polarized beams.

In the set-up shown in Fig. 5 in addition to the half wave phase plate we place a quarter wave phase plate at each arm of the interferometer,

$P P_{\lambda / 4,1}=\left(\begin{array}{ll}1 & 0 \\ 0 & i\end{array}\right)$

$P P_{\lambda / 4,2}=\left(\begin{array}{cc}1 & 0 \\ 0 & -i\end{array}\right)$

obtaining the following fields at the end of each arm:

$\mathbf{E}_{1}^{\prime \prime}(\mathbf{r})=\frac{f(\mathbf{r})}{\sqrt{2}}\left(\begin{array}{c}\cos \theta \\ i \sin \theta\end{array}\right)$,
$\mathbf{E}_{2}^{\prime \prime}(\mathbf{r})=\frac{f(\mathbf{r})}{\sqrt{2}}\left(\begin{array}{c}i \sin \theta \\ \cos \theta\end{array}\right)$.

After combining both beams at the output of the interferometer we have

$\mathbf{E}_{o}(\mathbf{r})=\frac{f(\mathbf{r})}{2} e^{i \theta}\left(\begin{array}{l}1 \\ 1\end{array}\right)$

This is a uniformly and totally polarized beam with only orbital angular momentum contribution

$j_{o}^{L}=+\hbar$,

$j_{0}^{S}=0$.

It should be noticed that we have given two examples, but a variety of possibilities can be obtained by varying the phase plates in both interferometer arms.

\section{Conclusions}

We have proposed different arrangements to generate orbital or/ and spin angular momentum in a light beam. The methods are mainly based on using non-uniformly polarized fields. By means of unconventional optical elements, such as azimuthal polarizers or polarization converters, we have found that we can generate fields with both uniform and non-uniform polarization across their transverse section and provide them with orbital and/or spin angular momentum. In this way, by choosing the appropriate setting, that could include combinations of the proposed arrangements, it is possible to synthesize a beam with the desired angular momentum. It should be noticed that in the proposed schemes the maximum orbital angular momentum that can be achieved is $|\hbar|$, due to the fact that we are using polarization as an intermediate step.

\section{Acknowledgments}

This work has been supported by the Ministerio de Ciencia e Innovación of Spain, project FIS2010-17543.

\section{References}

[1] T. Kuga, Y. Torii, N. Shiokawa, T. Hirano, Physical Review Letters 78 (1997) 4713.

[2] L. Paterson, M.P. MacDonald, J. Arlt, W. Sibbett, P.E. Bryant, K. Dholakia, Science $292(2001) 912$.

[3] G. Gibson, J. Courtial, M.J. Padgett, M. Vasnetsov, V. Pas'ko, S.M. Barnett, S. Franke-Arnold, Optics Express 12 (2004) 5448.

[4] C. Rotschild, S. Zommer, S. Moed, O. Hershcovit, S.G. Lipson, Applied Optics 43 (2004) 2397.

[5] G. Molina-Terriza, J.P. Torres, L. Torner, Nature Physics 3 (2007) 305

[6] S. Franke-Arnold, L. Allen, M. Padgett, Laser and Photonics Review 2 (2008) 299.

[7] L. Marrucci, C. Manzo, D. Paparo, Physical Review Letters 96 (2006) 163905.

[8] L. Marrucci, E. Karimi, S. Slussarenko, B. Piccirillo, E. Santamato, E. Nagali, F. Sciarrino, Journal of Optics 13 (2011) 064001.

[9] Y. Zhao, et al., Physical Review Letters 99 (2007) 073901.

[10] I. Mokhun, R. Brandel, Ju. Viktorovskaya, Ukraine Journal of Physiological Optics 7 (2006) 63.

[11] I. Mokhun, in: O.V. Angelsky (Ed.), Optical Correlation Techniques and Applications, Chapter 1, SPIE Press, Bellingham, Washington, USA, 2007, p. 1.

[12] I. Mokhun, R. Khrobatin, Journal of Optics. A-Pure Applied Optics 10 (2008) 064015.

[13] A. Bekshaev, M. Soskin, M. Vasnetsov, Paraxial Light Beams with Angular Momentum, Nova Science Publishers, New York, 2008.

[14] S. Lavi, R. Prochaska, E. Keren, Applied Optics 27 (1988) 3696.

[15] M.J. Bastiaans, Optik 82 (1989) 173.

[16] A.E. Siegman, Physical Society Photo-Optics Institute 1224 (1990) 2.

[17] J.Serna, R. Martínez-Herrero, P.M. Mejías, Journal of the Optical Society of America. A 8 (1991) 1094.

[18] J. Serna, J.M. Movilla, Optics Letters 26 (2001) 405.

[19] J. Serna, G. Piquero, Optics Communications 282 (2009) 1973.

[20] R. Martínez-Herrero, P.M. Mejías, G. Piquero, Springer Series in Optical Sciences, 147, 2009, Berlin.

[21] W.M. Lee, X.C. Yuan, K. Dholakia, Optics Communications 239 (2004) 129.

[22] J. Leach, E. Yao, M.J. Padgett, New Journal of Physics 6 (2004) 71.

[23] N.R. Heckenberg, R. McDuff, C.P. Smith, A.G. White, Optics Letters 17 (1992) 221.

[24] M.W. Beijersbergen, R.P.C. Coerwinkel, M. Kristensen, J.P. Woerdman, Optics Communications 112 (1994) 321.

[25] J.E. Curtis, B.A. Koss, D.G. Grier, Optics Communications 207 (2002) 169.

[26] S.S.R. Oemrawsingh, J.A.W. van Houwelingen, E.R. Eliel, J.P. Woerdman, E.J.K. Verstegen, J.G. Kloosterboer, G.W. 't Hooft, Applied Optics 43 (2004) 688.

[27] A. Niv, G. Biener, V. Kleiner, E. Hasman, Optics Communications 251 (2005) 306.

[28] V. Ramírez-Sánchez, G. Piquero, M. Santarsiero, Journal of Optics. A-Pure Applied Optics 11 (2009) 085708.

[29] M. Stalder, M. Schadt, Optics Letters 21 (1996) 1948. 